University of Windsor

Scholarship at UWindsor

Summer 2009

\title{
A matrix approach to status quo analysis in the graph model for conflict resolution
}

Haiyan Xu

Kevin W. Li Dr.

University of Windsor

Keith W. Hipel

D. Marc Kilgour

Follow this and additional works at: https://scholar.uwindsor.ca/odettepub

Part of the Business Commons

\section{Recommended Citation}

Xu, Haiyan; Li, Kevin W. Dr.; Hipel, Keith W.; and Kilgour, D. Marc. (2009). A matrix approach to status quo analysis in the graph model for conflict resolution. Applied Mathematics and Computation, 212 (2), 470-480.

https://scholar.uwindsor.ca/odettepub/66

This Article is brought to you for free and open access by the Odette School of Business at Scholarship at UWindsor. It has been accepted for inclusion in Odette School of Business Publications by an authorized administrator of Scholarship at UWindsor. For more information, please contact scholarship@uwindsor.ca. 
Elsevier Editorial System(tm) for Applied Mathematics and Computation Manuscript Draft

Manuscript Number: AMC-D-08-00620R1

Title: A Matrix Approach to Status Quo Analysis in the Graph Model for Conflict Resolution

Article Type: Full Length Article

Keywords: Status quo analysis; Decision support system; Matrix representation; Graph model for conflict resolution; Multiple decision makers

Corresponding Author: Professor Keith William Hipel, Ph.D.

Corresponding Author's Institution: University of Waterloo

First Author: Haiyan Xu, Msci.

Order of Authors: Haiyan Xu, Msci.; Kevin W Li, Ph.D.; Keith W Hipel, PhD; D. Marc Kilgour, Ph.D.

Abstract: An algebraic method is developed to carry out status quo analysis within the framework of the graph model for conflict resolution. As a post-stability analysis, the status quo analysis aims at assessing whether predicted equilibria are reachable from the status quo or any other initial state. Although pseudo-codes for status quo analysis have been developed, they are not yet implemented into a decision support system for use in practical applications. On the contrary, the novel matrix approach to status quo analysis designed here is convenient for computer implementations and an application to a real- orld conflict case study demonstrates its ease of employment. Moveover, the proposed explicit matrix approach reveals an inherent link between status quo analysis and the traditional stability analysis and, hence, provides the possibility of establishing an integrated paradigm for stability and status quo analyses. 
February 12, 2009

Melvin Scott, PhD

Editor-in-Chief

Applied Mathematics and Computation

494 Carlton Court

Ocean Isle Beach, NC 28469

USA

Email: MScott8223@atmc.net

Re: Revision for Ms. Ref. \#: AMC-D-08-00620

Dear Dr. Scott:

Thank you for kindly forwarding us the reviewer's comments on our submission entitled "A Matrix Approach to Status Quo Analysis in the Graph Model for Conflict Resolution” (Paper ID\#: AMC-D-08-00620) by H. Xu, K.W. Li, K.W. Hipel and D.M. Kilgour. This revision has incorporated the reviewer's thoughtful comments. For details, please refer to our response file as well as the revised manuscript.

Thank you, once again, for your time and effort in processing our article. I look forward to possibly seeing our paper appear in your fine journal.

Sincerely,

Keith W. Hipel, Ph.D., University Professor

Department of Systems Design Engineering

University of Waterloo

Waterloo, Ontario N2L 3G1

Canada

Email: kwhipel@uwaterloo.ca 


\title{
A Matrix Approach to Status Quo Analysis in the Graph Model for Conflict Resolution
}

\author{
Haiyan $\mathrm{Xu}^{a}$, Kevin W. Li ${ }^{b}$, Keith W. Hipel ${ }^{a *}$, and D. Marc Kilgour ${ }^{c}$ \\ ${ }^{a}$ Department of Systems Design Engineering, University of Waterloo, Waterloo, \\ Ontario, N2L 3G1, Canada \\ ${ }^{b}$ Odette School of Business, University of Windsor, Windsor, Ontario, Canada \\ ${ }^{c}$ Department of Mathematics, Wilfrid Laurier University, Waterloo, Ontario, Canada
}

\begin{abstract}
An algebraic method is developed to carry out status quo analysis within the framework of the graph model for conflict resolution. As a post-stability analysis, the status quo analysis aims at assessing whether predicted equilibria are reachable from the status quo or any other initial state. Although pseudo-codes for status quo analysis have been developed, they are not yet implemented into a decision support system for use in practical applications. On the contrary, the novel matrix approach to status quo analysis designed here is convenient for computer implementations and an application to a real-world conflict case study demonstrates its ease of employment. Moveover, the proposed explicit matrix approach reveals an inherent link between status quo analysis and the traditional stability analysis and, hence, provides the possibility of establishing an integrated paradigm for stability and status quo analyses.
\end{abstract}

Key words: Status quo analysis; Decision support system; Matrix representation; Graph model for conflict resolution; Multiple decision makers

\section{Introduction}

Conflict arises whenever multiple stakeholders with diverse interests interact with each other [5]. It is common to observe conflicts across a wide range of scales and settings. Family members may argue over where to spend their next vacation. In the marketplace, fierce competitions for market shares are accelerating across all industries with the globalization of world economies. Governments account for many conflicts. For example, it is a tradition for the Canadian federal and provincial governments to argue over how medicare should be funded and spent.

* Corresponding author. E-mail:kwhipel@uwaterloo.ca; Fax:1-519-7464791 (K.W. Hipel). 
The ubiquity of conflicts creates a great need for formal methodologies to "assist in the understanding, modeling, and analysis of conflict" [7]. Conflict resolution has been investigated within many different disciplines [13], such as international studies, political science, psychology and law $[2,3,6]$, as well as from mathematical and engineering perspectives $[1,7,14,15,21,22]$.

Among these formal methodologies to handle strategic conflict, the Graph Model for Conflict Resolution (GMCR) $[7,16]$ is probably the most simple but flexible framework. A graph model for a strategic conflict is comprised of a finite set of decision makers (DMs), $N$, a set of feasible states, $S$, a preference relation $\succeq_{i}$ on $S$ for each DM $i$, and a directed graph $G_{i}=\left\{S, A_{i}\right\}$. In each directed graph, $S$ is the vertex set, and each oriented arc in $A_{i} \subseteq S \times S$ indicates that DM $i$ can make a one-step unilateral move from the initial state to the terminal state of the arc. A decision support system (DSS), GMCR II, implements the methodology of the graph model and facilitates the stability analysis of conflict models $[8,9,12]$. Stability analysis determines for each state its individual stability and group stability for all DMs (equilibrium) under appropriate stability definitions (solution concepts), where equilibria often correspond to potential resolutions of the conflict. In 1989, Wang et al. [27] defined solution concepts in Hypergames. Recently, Hamouda et al. [10] proposed new solution concepts that take strength of preference into account.

When a conflict is modeled as a graph model, a point in time has to be selected first and the current (or initial) state of the conflict is referred to as the status quo. When the stability of a state is assessed in the stability analysis stage, it is not a concern whether this state is actually achievable from the status quo state. As a follow-up analysis, status quo analysis is designed to trace the evolution of a conflict from the status quo state to any desirable outcome. Specifically, it usually focuses on examining whether predicted equilibria are reachable from the status quo, and if so, how to reach them. Thus, status quo analysis provides useful dynamic and forward-looking insights into a strategic conflict $[18,19]$.

In the original graph model, the stability analysis is carried out within a well-designed logic structure [7]. When status quo analysis algorithms were developed by Li et al. [18-20], this line of thinking was retained and pseudo-codes were furnished following a similar logic structure. A drawback of this structure is that it is difficult to maintain the existing DSS GMCR II and to extend it to incorporate new developments such as status quo analysis. To overcome this challenge, Xu et al. [24,25] developed an innovative matrix system to represent DMs' preferences and interactions in a graph model. The matrix representation effectively converts the stability analysis from a logic structure to an algebraic system: when a graph model is appropriately coded into matrices, stability analyses can be expeditiously completed by a series of matrix operations. It is obvious that this algebraic structure is flexible and can be easily modified to handle large-scale graph models.

Due to the difficulty in integrating status quo analysis into the DSS GMCR II and 
the ease of implementing the matrix representation of stability analysis, it is natural to exploit the matrix approach to perform status quo analysis. This paper reports the recent development of an algebraic method for status quo analysis within the graph model paradigm. Specifically, a graph model is first expressed as matrices and status quo analysis is formulated as a series of matrix operations. The proposed matrix approach to status quo analysis is not only kept consistent with matrix representations of stability analysis, but also further reveals the relationship between status quo and stability analyses. The novel algebraic method facilitates the development of improved algorithms to implement status quo analysis in a DSS. Additionally, due to the nature of the explicit algebraic expressions, the proposed method is more effective, convenient, flexible, and extendable for carrying out status quo analysis, compared with the existing methods. The effectiveness of this matrix approach is demonstrated by a real-world case study, the Elmira conflict over groundwater contamination.

The rest of the paper is organized as follows. Section 2 provides the structure of matrix representation for status quo analysis. The proposed approach and main results are presented in Section 3. In Section 4, a case study of the Elmira conflict, which took place in southwestern Ontario, Canada, is carried out to illustrate how the proposed method can be applied in practice and demonstrate the unique features of the algebraic method. The paper concludes with some comments in Section 5.

\section{The Basic Structure of Matrix Representations}

To discuss the representation of a graph model using matrices, some preliminaries on the graph model and status quo analysis have to be introduced first.

\subsection{The Components of a Graph Model}

In a graph model, the preferences of DM $i$ over the set of states $S$ can be expressed by a pair of binary relations $\left\{\succ_{i}, \sim_{i}\right\}$ on $S$, where $a \succ_{i} b$ indicates that DM $i$ prefers $a$ to $b$, and $a \sim_{i} b$ means that DM $i$ is indifferent between $a$ and $b$ (or equally prefers $a$ and $b$ ). Also, $a \succeq_{i} b$ means either $a \succ_{i} b$ or $a \sim_{i} b$. It is assumed that the preference relations of each DM $i \in N$ have following properties [7]:

(i) $\succ_{i}$ is asymmetric.

(ii) $\sim_{i}$ is reflexive and symmetric.

(iii) $\left\{\succ_{i}, \sim_{i}\right\}$ is strongly complete.

In the graph model, preference relationships play an important role in determining the 
stability status of a state. Preference information can be either transitive or intransitive. For $a, b, c \in S$, if $a \succ b$ and $b \succ c$ imply $a \succ c$, then the preference $\succ$ is transitive. Otherwise, preferences are intransitive. Note that the graph model methodology itself does not require preferences to be transitive though the current computer implementation GMCR II does due to its inherent preference elicitation structure. A distinct advantage of the matrix representation for stability analysis is that it accommodates both transitive and intransitive preferences. As the development of this article reveals, this advantage has been retained for status quo analysis.

The preference information may be given as ordinal preferences (ranking of states from most to least preferred, with ties allowed), cardinal preferences (the preference of each state is determined by a real-valued preference function), or relative preferences (intransitive preferences may be present) used by the graph model for each DM. When preferences are not provided as relative preferences, they will be converted first before the preference information is fed into a graph model. For a cardinal preference relation, assume that $p_{i}(s)$ is a preference function over $s \in S$ for DM $i$ and rank the states in $S$ as: $1, s_{1} ; 2, s_{2} ; \cdots ; m, s_{m}(\mathrm{~m}=|S|$, the number of states in $S)$; then let $p_{i}\left(s_{k}\right)=p_{i}(k)$. For an ordinal preference, one may define its preference function by

$$
p_{i}(s)=\left|\left\{x \in S: s \succeq_{i} x\right\}\right|, \forall s \in S, \text { for } i=1,2, \cdots, n,
$$

where $x$ is a dummy variable and $|U|$ denotes the cardinality of the set $U=\{x \in S$ : $\left.s \succeq_{i} x\right\}$.

$R_{i}(s)$ is DM $i^{\prime} s$ reachable list from state $s$, containing all states to which DM $i$ can unilaterally move from state $s$ in one step. Algebraically, $R_{i}(s)=\left\{q \in S:(s, q) \in A_{i}\right\}$, representing DM $i^{\prime} s$ unilateral moves (UMs). For DM $i$, a unilateral improvement (UI) from state $s$ is defined by $R_{i}^{+}(s)=\left\{q \in S:(s, q) \in A_{i}\right.$ and $\left.q \succ_{i} s\right\}$ [7].

A legal sequence of UMs is a sequence of states linked by unilateral moves by members of a subset of DMs, in which any DM may move more than once, but not twice consecutively. Let $H \subseteq N$ be any subset of the DMs, then $R_{H}(s) \subseteq S$ denotes the set of states that can be reached by any legal sequence of UMs, by some or all DMs in $H$, starting from state $s$. A legal sequence of UIs can be defined similarly. $R_{H}^{+}(s) \subseteq S$ indicates the set of all states that result from any legal sequence of UIs, starting from state $s . R_{H}(s)$ and $R_{H}^{+}(s)$ are formally defined by Fang et al. [7].

If, for any sequence of two consecutive UMs by DM $i$, there always exists an equivalent single move, then DM $i^{\prime}$ s graph is transitive. When all DMs' graphs are transitive, the requirement of no consecutive moves becomes irrelevant. 


\subsection{Notation for Status Quo Analysis in the Graph Model}

Some notation about status quo analysis in the graph model is introduced as follows:

(i) $S Q$ denotes the status quo state.

(ii) $S_{i}^{(k)}(s)$ and $S_{i}^{(k,+)}(s)$ indicate all states reachable from $S Q=s$ in exactly $k(k \geq 1)$ legal UMs and UIs, respectively, with last mover DM $i$.

(iii) $V^{(k)}(s)$ and $V^{(k,+)}(s)$ contain all states reachable from $S Q=s$ in at most $k(k \geq 1)$ legal UMs and UIs, respectively. $V_{i}^{(k)}(s)$ and $V_{i}^{(k,+)}(s)$ consist of all states reachable from $S Q=s$ in at most $k(k \geq 1)$ legal UMs and UIs, respectively, with last mover $\mathrm{DM} i$. i.e., $V_{i}^{(k)}(s)=S_{i}^{(k)}(s) \cup V^{(k-1)}(s)$ and $V_{i}^{(k,+)}(s)=S_{i}^{(k,+)}(s) \cup V^{(k-1,+)}(s)$.

(iv) $A_{i}^{(k)}(s)$ and $A_{i}^{(k,+)}(s)$ include all arcs with last mover DM $i$ in sequences of at most $k(k>1)$ legal UMs and UIs, respectively, from $S Q=s$.

Note: Similar to the concepts of reachable list $R_{i}(s)$ and UI list $R_{i}^{+}(s)$, tracking the states that can be attained from $s$ in one-step UM and UI, respectively, we introduce the notation $A_{i}(s)$ and $A_{i}^{+}(s)$ to denote the sets of arcs by DM $i$ in one-step UMs and UIs from state $s$, respectively. Along this line, all UM and UI arcs can be expressed as $A_{i}=\bigcup_{s \in S} A_{i}(s)$ and $A_{i}^{+}=\bigcup_{s \in S} A_{i}^{+}(s)$. (U denotes the union operation, e.g., $\bigcup_{s \in S} A_{i}(s)=$ $\left.\left\{A_{i}(s): \forall s \in S\right\}\right)$. Let $A=\bigcup_{i \in N} A_{i}$. In our aforesaid notation, the superscript $k$ refers to the iteration step when the arc sets are progressively expanded.

\subsection{Components of Matrix Representations}

Now, we can introduce basic components of matrix representations and explain how relevant matrices are constructed.

\subsubsection{Unilateral Move and Unilateral Improvement Matrices}

Let $m=|S|$. $W=M \circ G$ is defined as the Hadamard product of two $m \times m$ matrices $M$ and $G$, i.e., if $M(s, q)$ and $G(s, q)$ denote the $(s, q)$ entries of matrices $M$ and $G$, respectively, then the $m \times m$ matrix $W$ has its $(s, q)$ entry $W(s, q)=M(s, q) \cdot G(s, q)$. Additionally, $Q=M \vee G$ is defined as the $m \times m$ matrix with its $(s, q)$ entry

$$
Q(s, q)=\left\{\begin{array}{l}
1 \text { if } M(s, q)+G(s, q) \neq 0, \\
0 \text { otherwise. }
\end{array}\right.
$$


A sign function, $\operatorname{sign}(\cdot)$, maps an $m \times m$ matrix with its $(s, q)$ entry $M(s, q)$ to another $m \times m$ matrix

$$
\operatorname{sign}[M(s, q)]=\left\{\begin{array}{lc}
1 & M(s, q)>0, \\
0 & M(s, q)=0, \\
-1 & M(s, q)<0 .
\end{array}\right.
$$

Let $i \in N, s \in S$, and $m=|S|$. An $m \times m$ 0-1 matrix $J_{i}$ is defined by

$$
J_{i}(s, q)=\left\{\begin{array}{l}
1 \text { if }(s, q) \in A_{i}, \\
0 \text { otherwise }
\end{array}\right.
$$

It is clear that $R_{i}(s)=\left\{q: J_{i}(s, q)=1\right\}$, so $J_{i}$ is called DM $i^{\prime} s$ unilateral move (UM) matrix. For DM $i$, a unilateral improvement (UI) matrix $J_{i}^{+}$is defined by

$$
J_{i}^{+}(s, q)=\left\{\begin{array}{l}
1 \text { if } J_{i}(s, q)=1 \text { and } q \succ_{i} s \\
0 \text { otherwise }
\end{array}\right.
$$

Obviously, $R_{i}^{+}(s)=\left\{q: J_{i}^{+}(s, q)=1\right\}$.

If $R_{i}(s)$ and $R_{i}^{+}(s)$ are written as 0-1 row vectors where one or zero in the $q$ th entry indicates that state $q$ is reachable or not reachable from state $s$ in a one-step UM and UI, respectively, then

$$
R_{i}(s)=e_{s}^{T} \cdot J_{i} \text { and } R_{i}^{+}(s)=e_{s}^{T} \cdot J_{i}^{+},
$$

where $e_{s}^{T}$ denotes the transpose of the $s^{\text {th }}$ standard basis vector of the $m$-dimensional Euclidean space. UM and UI matrices, $J_{i}$ and $J_{i}^{+}$, depict unilateral moves and unilateral improvements in one step by DM $i$ among a set of states.

For relative preferences, the preference matrix $P_{i}^{+}$for DM $i$ is defined by

$$
P_{i}^{+}(s, q)=\left\{\begin{array}{l}
1 \text { if } q \succ_{i} s \\
0 \text { otherwise }
\end{array}\right.
$$

For ordinal or cardinal preferences, after they are converted to relative preferences as per the preference function $p_{i}(s)$ defined by equation (1) in Section 2.1, the preference 
matrix for DM $i$ can be constructed by

$$
P_{i}^{+}(s, q)=\operatorname{sign}\left(\frac{a b s\left(p_{i}(q)-p_{i}(s)\right)+p_{i}(q)-p_{i}(s)}{2}\right),
$$

where $\operatorname{abs}\left(p_{i}(q)-p_{i}(s)\right)$ denotes the absolute value of function $p_{i}(q)-p_{i}(s)$. It is clear that the relationships among the UM matrix, UI matrix, and preference matrix are established as follows:

$$
J_{i}^{+}(s, q)=J_{i}(s, q) \circ P_{i}^{+}(s, q) .
$$

\subsubsection{Joint Move and Joint Improvement Matrices}

Let $H \subseteq N$. We now demonstrate how to find matrices corresponding to $R_{H}(s)$, the set of reachable states via joint UMs by $H$ from $s$, and $R_{H}^{+}(s)$, the set of reachable states via joint UIs by $H$ from $s$, for all $s \in S$. First, define two $m \times m$ matrices $M_{i}^{(t)}$ and $M_{i}^{(t,+)}$ with $(s, q)$ entries as follows:

Definition 1 For $i \in N$ and $t=1,2,3, \cdots$,

$$
M_{i}^{(t)}(s, q)=\left\{\begin{array}{l}
1 \text { if } q \in S \text { is reachable from } s \in S \text { in } \\
\quad \text { exactly } t \text { legal UMs with last mover DM } i \\
0 \text { otherwise, }
\end{array}\right.
$$

and

$$
M_{i}^{(t,+)}(s, q)=\left\{\begin{array}{l}
1 \text { if } q \in S \text { is reachable from } s \in S \text { in } \\
\quad \text { exactly } t \text { legal UIs with last mover DM } i \\
0 \text { otherwise. }
\end{array}\right.
$$

Note that $M_{i}^{(t)}$ and $M_{i}^{(t,+)}$ defined here are different from the matrices $M_{i}^{t}$ and $M_{i}^{t,+}$ defined by [26] in which $i$ denotes the first mover, whereas in $M_{i}^{(t)}$ and $M_{i}^{(t,+)}$ given here, $i$ is the last mover. However, the final outcomes are obviously related. Now we have two ways to construct joint matrices by tracking the first or last mover, demonstrating the flexibility of the proposed matrix method.

Let $n=|N|$ denote the number of DMs in the set $N$. In order to describe possible moves in the graph model with $n$ DMs using matrix representations, define two more $m \times m$ matrices, a joint UM matrix, $M_{H}$, and a joint UI matrix, $M_{H}^{+}$, corresponding to the joint sets, $R_{H}(s)$ and $R_{H}^{+}(s)$, for all $s \in S$, respectively, as follows: 


\section{Definition 2}

$$
M_{H}(s, q)=\left\{\begin{array}{l}
1 \text { if } q \in R_{H}(s), \\
0 \text { otherwise }
\end{array} \quad \text { and } \quad M_{H}^{+}(s, q)=\left\{\begin{array}{l}
1 \text { if } q \in R_{H}^{+}(s) \\
0 \text { otherwise }
\end{array}\right.\right.
$$

With the introduction of these matrices, we are in a position to elaborate the matrix approach to status quo analysis.

\section{Matrix Representations of Status Quo Analysis}

To facilitate the development of the matrix approach, existing status quo analysis algorithms are now introduced.

\subsection{Existing Work on Status Quo Analysis}

Two pseudo-codes for status quo analysis that allow for unilateral moves and unilateral improvements, respectively, are provided by Li et al. [19]. In status quo analysis, if a DM moves twice in succession, the consecutive moves are deemed illegal.

The following pseudo-code allows any unilateral moves:

Pseudo-code for status quo analysis with any UMs (Adapted from [19])

1. Let $h=0, S_{i}^{(0)}(S Q)=\{S Q\}, V^{(0)}(S Q)=\{S Q\}$, and $A_{i}^{(0)}(S Q)=\emptyset($ for $i \in N)$

2. Let $h=h+1$, and for each $i \in N$, update $S_{i}^{(h)}(S Q)$ and $A_{i}^{(h)}(S Q)$ as:

$$
\left.\begin{array}{rl}
S_{i}^{(h)}(S Q) & =\bigcup\left\{R_{i}(s): s \in \bigcup_{j \in N-\{i\}} S_{j}^{(h-1)}(S Q)\right\} \\
A_{i}^{(h)}(S Q) & =\left\{\begin{array}{l}
A_{i}^{(h-1)}(S Q) \\
A_{i}^{(h-1)}(S Q) \bigcup\left\{\left(s, s^{\prime}\right): s \in \bigcup_{j \in N-\{i\}} S_{i}^{(h-1)}(S Q) \text { and } s^{\prime} \in R_{i}(s)\right\} \text { otherwise. }
\end{array}\right. \\
V^{(h)}(S Q) & =\left(\bigcup_{i \in N} S_{i}^{(h)}(S Q)\right) \bigcup V^{(h-1)}(S Q)
\end{array}\right\}
$$

Otherwise, go to 2 .

Although Li et al. [19] indicate that the process must stop in a finite number of itera- 
tions, they have not explained why this is so in detail. Let $L_{1}=\left|\bigcup_{i \in N} A_{i}\right|$, the number of arcs in all DMs' graphs. One can prove that the process must stop in less than $L_{1}$ iterations. If the process terminates at step $k$, the status quo diagram of permitting any UMs in the graph model is given by $\left(V^{(k)}(S Q), \bigcup_{i \in N} A_{i}^{(k)}(S Q)\right)$.

Similarly, the pseudo-code of allowing unilateral improvements only is provided as follows:

Pseudo-code for status quo analysis with UIs only (Adapted from $[18,19]$ )

1. Let $h=0, S_{i}^{(0,+)}(S Q)=\{S Q\}, V^{(0,+)}(S Q)=\{S Q\}$, and $A_{i}^{(0,+)}(S Q)=\emptyset($ for $i \in N)$

2. Let $h=h+1$, and for each $i \in N$, update $S_{i}^{(h,+)}(S Q), A_{i}^{(h,+)}(S Q)$ and $V^{(h,+)}(S Q)$ as:

$$
\begin{aligned}
& S_{i}^{(h,+)}(S Q)=\bigcup\left\{R_{i}^{+}(s): s \in \bigcup_{j \in N-\{i\}} S_{j}^{(h-1,+)}(S Q)\right\} \\
& A_{i}^{(h,+)}(S Q)=\left\{\begin{array}{l}
A_{i}^{(h-1,+)}(S Q) \\
A_{i}^{(h-1,+)}(S Q) \bigcup\left\{\left(s, s^{\prime}\right): s \in \bigcup_{j \in N-\{i\}} S_{i}^{(h-1,+)}(S Q), s^{\prime} \in R_{i}^{+}(s)\right\} \text { otherwise. }
\end{array}\right. \\
& \qquad V^{(h,+)}(S Q)=\left(\bigcup_{i \in N} S_{i}^{(h,+)}(S Q)\right) \bigcup V^{(h-1,+)}(S Q) \\
& \text { 3. If } \bigcup_{i \in N} A_{i}^{(h,+)}(S Q)=\bigcup_{i \in N} A_{i}^{(h-1,+)}(S Q), \text { stop. }
\end{aligned}
$$

Otherwise, go to 2.

Similarly, $\left(V^{(k,+)}(S Q), \bigcup_{i \in N} A_{i}^{(k,+)}(S Q)\right)$ determines the status quo diagram allowing UIs only when the aforesaid process stops at iteration step $k$.

Using status quo diagrams, significant information about the conflict under investigation can be obtained. Specifically, if an equilibrium appears in the diagram, it exists at least one path from the status quo to the equilibrium. By tracing the status quo diagram, one can determine a viable evolution path from the status quo state to this equilibrium. Conversely, if a predicted equilibrium does not appear in the status quo diagram, there is no way for the conflict to settle at this equilibrium as it is not reachable from the status quo [19]. This useful information cannot be obtained by using GMCR II since the aforementioned status quo analysis algorithms have not been integrated into the DSS. To garnish these additional insights, analysts have to rely on tedious manual calculations. The matrix approach developed in the next subsection aims at furnishing a flexible and easy-to-implement procedure for carrying out status quo analysis. Analysts with basic matrix operations knowledge will be able to capitalize on the procedure to conduct a status quo analysis. 


\subsection{A Matrix Approach to Status Quo Analysis}

Based on Definition 1, we have:

Lemma 1 The two $m \times m$ matrices $M_{i}^{(t)}$ and $M_{i}^{(t,+)}$ can be expressed inductively by

$$
M_{i}^{(t)}=\operatorname{sign}\left[\left(\bigvee_{j \in H-\{i\}} M_{j}^{(t-1)}\right) \cdot J_{i}\right] \text {, with } M_{i}^{(1)}=J_{i},
$$

and

$$
M_{i}^{(t,+)}=\operatorname{sign}\left[\left(\bigvee_{j \in H-\{i\}} M_{j}^{(t-1,+)}\right) \cdot J_{i}^{+}\right] \text {, with } M_{i}^{(1,+)}=J_{i}^{+} .
$$

Proof: Although matrix $M_{i}^{(t)}$ defined here is different from the matrix $M_{i}^{t}$ [26], the proof of (4) can be carried out in a similar fashion as that given by $\mathrm{Xu}$ et al. [26]. Consequently, we only verify (5) here. For $t=2$, assume that $G(s, q)$ denotes the $(s, q)$ entry of the matrix $G=\left(\underset{j \in H-\{i\}}{\bigvee} J_{j}^{+}\right) \cdot J_{i}^{+}$. Based on the definition of matrix multiplication, $G(s, q) \neq 0$ iff state $q$ is reachable from state $s$ in exactly two unilateral improvements with last mover DM $i$. The condition $j \in H-\{i\}$ implies that DM $i$ does not make two moves consecutively. Hence, $G(s, q) \neq 0$ iff state $q$ is reachable from state $s$ in exactly 2 legal UIs. Then, Definition 1 for $t=2$ implies that

$$
\operatorname{sign}\left[\left(\bigvee_{j \in H-\{i\}} J_{j}^{+}\right) \cdot J_{i}^{+}\right]=\operatorname{sign}\left[\left(\bigvee_{j \in H-\{i\}} M_{j}^{(1,+)}\right) \cdot J_{i}^{+}\right]=M_{i}^{(2,+)} .
$$

It is easy to extend this result to general values of $t$. Assume that $Q(s, q)$ is the $(s, q)$

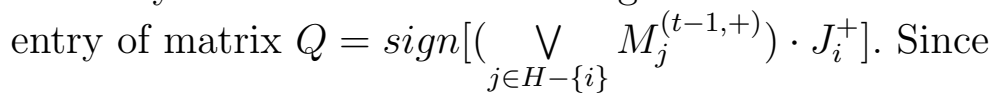

$$
M_{j}^{(t-1,+)}(s, q)=\left\{\begin{array}{c}
1 \text { if } q \in S \text { is reachable from } s \in S \text { in exactly } \\
t-1 \text { legal UIs with last mover DM } j, \\
0 \text { otherwise, }
\end{array}\right.
$$

then by matrix multiplication,

$$
Q(s, q)=\left\{\begin{array}{c}
1 \text { if } q \in S \text { is reachable from } s \in S \text { in } \\
\quad \text { exactly } t \text { legal UIs with last mover DMi, } \\
0 \text { otherwise. }
\end{array}\right.
$$


Hence $Q=M_{i}^{(t,+)}$ based on Definition 1. i.e., equation (5) holds.

Next we define two status quo analysis matrices $M_{i}^{S Q^{(t)}}$ and $M_{i}^{S Q^{(t,+)}}$ to trace the evolution of a conflict from a status quo state to any desirable outcome as follows:

Definition 3 For $i \in N$ and $t=1,2,3, \cdots$,

$$
M_{i}^{S Q^{(t)}}(s, q)=\left\{\begin{array}{l}
1 \text { if } q \in V_{i}^{(t)}(s), \\
0 \text { otherwise, }
\end{array} \quad \text { and } M_{i}^{S Q^{(t,+)}}(s, q)=\left\{\begin{array}{l}
1 \text { if } q \in V_{i}^{(t,+)}(s) \\
0 \text { otherwise }
\end{array}\right.\right.
$$

Specifically, $M_{i}^{S Q^{(t)}}(s, q)=1$ and $M_{i}^{S Q^{(t,+)}}(s, q)=1$ denote that state $q$ is reachable from status quo state $s$ in at most $t$ UMs and UIs, respectively, with last mover $i$. Based on Definitions 1 and 3, Theorem 1 can be easily derived.

Theorem 1 Let $s \in S, H \subseteq N$, and $H \neq \emptyset$. Then status quo analysis matrices $M_{i}^{S Q^{(k)}}$ and $M_{i}^{S Q^{(k,+)}}$ can be respectively expressed as

$$
M_{i}^{S Q^{(k)}}=\bigvee_{t=1}^{k} M_{i}^{(t)} \quad \text { and } \quad M_{i}^{S Q^{(k,+)}}=\bigvee_{t=1}^{k} M_{i}^{(t,+)}
$$

Proof: We prove that $M_{i}^{S Q^{(k,+)}}=\bigvee_{t=1}^{k} M_{i}^{(t,+)}$. Assume that $M_{i}^{S Q^{(k,+)}}(s, q)$ denotes the $(s, q)$ entry of the matrix $M_{i}^{S Q^{(k,+)}}$. Based on Definition $3, M_{i}^{S Q^{(k,+)}}(s, q)=1$ iff $q \in$ $V_{i}^{(t,+)}(s)$. i.e., $q$ is reachable from $S Q=s$ in at most $k$ legal UIs, with last mover DM $i$.

Let $\bigvee_{t=1}^{k} M_{i}^{(t,+)}(s, q)$ denote the $(s, q)$ entry of the matrix $\bigvee_{t=1}^{k} M_{i}^{(t,+)}$. By Definition 1 , $\bigvee_{t=1}^{k} M_{i}^{(t,+)}(s, q)=1$ iff $\exists 1 \leq l \leq k$, such that $M_{i}^{(l,+)}(s, q)=1$. i.e., $q$ is reachable from $\stackrel{t=1}{S Q}=s$ in exactly $l$ legal UIs, with last mover DM $i$. It implies that $q$ is reachable from $S Q=s$ in at most $k$ legal UIs, with last mover DM $i$. Consequently, $\bigvee_{t=1}^{k} M_{i}^{(t,+)}(s, q)=1$ iff $M_{i}^{S Q^{(k,+)}}(s, q)=1$. Since $M_{i}^{S Q^{(k,+)}}$ and $\bigvee_{t=1}^{k} M_{i}^{(t,+)}$ are $m \times m 0$-1 matrices, it follows that $M_{i}^{S Q^{(k,+)}}=\bigvee_{t=1}^{k} M_{i}^{(t,+)}$.

$M_{i}^{S Q^{(k)}}=\bigvee_{t=1}^{k} M_{i}^{(t)}$ can be proven similarly.

Theorem 1 provides results equivalent to those from the two pseudo-codes. In fact, Theorem 1 is tested by using the Elmira model in Section 4 and identical results are obtained as those shown by Li et al. [19], but a distinct advantage of this approach is its ease to be implemented in any programming environment with matrix functionalities. 
Furthermore, this algebraic method also establishes the following inherent relationship between stability analysis and status quo analysis.

\subsection{Relationship Between Joint Matrices and Status Quo Analysis Matrices}

The current literature treats status quo analysis and stability analysis as two separate research topics. However, the matrix approach here reveals that these two analyses are inherently linked.

In $n$-DM models, $R_{H}$, the set of reachable states via joint unilateral moves by $H$, and $R_{H}^{+}$, the set of reachable states via joint unilateral improvements by $H$, are key components for stability analysis. Although Fang et al. [7] provide a set of pseudocodes for constructing $R_{H}$ and $R_{H}^{+}$, the process is complicated and hard to be extended or modified. Therefore, $\mathrm{Xu}$ et al. [26] develop two inductive procedures to construct joint matrices $M_{H}$ and $M_{H}^{+}$corresponding to joint sets $R_{H}$ and $R_{H}^{+}$. Subsequently, these joint matrices replace the two joint sets and play an important role in stability analysis. The following Lemma 2 asserts that if there does not exist any new appropriate arc in the graph model, then corresponding joint moves will stop.

Lemma 2 Let $S Q=s$ and $H \subseteq N$.

(1) If $\bigcup_{i \in H} A_{i}^{(k+1)}(s)=\bigcup_{i \in H} A_{i}^{(k)}(s)$, then $V^{(k+1)}(s)=V^{(k)}(s)$ and $R_{H}(s)=V^{(k)}(s)$;

(2) If $\bigcup_{i \in H} A_{i}^{(k+1,+)}(s)=\bigcup_{i \in H} A_{i}^{(k,+)}(s)$, then $V^{(k+1,+)}(s)=V^{(k,+)}(s)$ and $R_{H}^{+}(s)=V^{(k,+)}(s)$.

Proof: We prove (2) by contradiction. Assume that $\exists q \in V^{(k+1,+)}(s) \backslash V^{(k,+)}(s)$ but $\bigcup_{i \in H} A_{i}^{(k+1,+)}(s)=\bigcup_{i \in H} A_{i}^{(k,+)}(s)$. Since $V^{(k+1,+)}(s)=\left(\bigcup_{i \in H} S_{i}^{(k+1,+)}(s)\right) \cup V^{(k,+)}(s)$, then $\exists$ $i \in H$, such that $q \in S_{i}^{(k+1,+)}(s) \backslash V^{(k,+)}(s)$. Hence, $\exists s_{1} \in \bigcup_{j \in N-\{i\}} S_{j}^{(k,+)}(s)$ such that $q \in R_{i}^{+}\left(s_{1}\right)$. Clearly, this implies that $\left(s_{1}, q\right) \in A_{i}^{(k+1,+)}(s) \backslash \bigcup_{i \in H} A_{i}^{(k,+)}(s)$, contradicting with the hypothesis that $\bigcup_{i \in H} A_{i}^{(k+1,+)}(s)=\bigcup_{i \in H} A_{i}^{(k,+)}(s)$. Thus, $V^{(k+1,+)}(s)=V^{(k,+)}(s)$ when $\bigcup_{i \in H} A_{i}^{(k+1,+)}(s)=\bigcup_{i \in H} A_{i}^{(k,+)}(s)$. It is obvious that if $V^{(k+1,+)}(s)=V^{(k,+)}(s)$, then $\bigcup_{i \in H} S_{i}^{(k+1,+)}(s) \subseteq V^{(k,+)}(s)$. Consequently, there does not exist any new arc $A_{i}^{(k+1,+)}(s) \subseteq$ $A_{i}^{+}$, then corresponding joint unilateral improvements from state $s$ will stop at step $k$ , i.e., $R_{H}^{+}(s)=V^{(k,+)}(s)$. Result (1) can be proved in a similar manner.

For $H \subseteq N$, let $\left|\bigcup_{i \in H} A_{i}\right|$ and $\left|\bigcup_{i \in H} A_{i}^{+}\right|$denote the number of UM arcs and UI arcs, respectively, in $H$ 's graphs. Then the following Lemma 3 can be easily derived following Lemma 2.

Lemma 3 Let $\delta_{1}$ and $\delta_{2}$ denote the number of iterations required to find $R_{H}(s)$ and 
$R_{H}^{+}(s)$, respectively, for $\forall s \in S$. Then

$$
\delta_{1} \leq\left|\bigcup_{i \in H} A_{i}\right| \quad \text { and } \quad \delta_{2} \leq\left|\bigcup_{i \in H} A_{i}^{+}\right| .
$$

Proof: We prove $\delta_{2} \leq\left|\bigcup_{i \in H} A_{i}^{+}\right|$. From Lemma 2, if there does not exist any new UI arc, then the corresponding joint UI moves will stop. Consequently, the number of iterations to find joint UIs, $R_{H}^{+}(s)$, is less than the number of UI arcs in $H$ 's graphs. i.e., $\delta_{2} \leq\left|\bigcup_{i \in H} A_{i}^{+}\right|$.

Similarly, we can prove $\delta_{1} \leq\left|\bigcup_{i \in H} A_{i}\right|$.

Fix $H \subseteq N$. We now demonstrate how to find joint matrices corresponding to $R_{H}(s)$, the set of reachable states via joint UMs by $H$ from $s, R_{H}^{+}(s)$, the set of reachable states via joint UIs by $H$ from $s, s \in S$, respectively.

Let $L_{1}=\left|\bigcup_{i \in H} A_{i}\right|$ and $L_{2}=\left|\bigcup_{i \in H} A_{i}^{+}\right|$. Then, the following theorem can be derived using Lemma 3.

Theorem 2 Let $s \in S, H \subseteq N$, and $H \neq \emptyset$. Then joint $U M$ matrix $M_{H}$ and joint $U I$ matrix $M_{H}^{+}$can be respectively expressed by

$$
M_{H}=\bigvee_{t=1}^{L_{1}} \bigvee_{i \in H} M_{i}^{(t)} \text { and } M_{H}^{+}=\bigvee_{t=1}^{L_{2}} \bigvee_{i \in H} M_{i}^{(t,+)}
$$

Proof: The proof for $M_{H}=\bigvee_{t=1}^{L_{1}} \bigvee_{i \in H} M_{i}^{(t)}$ is similar to that shown by [26]. Now we prove $M_{H}^{+}=\bigvee_{t=1}^{L_{2}} \bigvee_{i \in H} M_{i}^{(t,+)}$. Assume that $G=\bigvee_{t=1}^{L_{2}} \bigvee_{i \in H} M_{i}^{(t,+)}$. Based on Definition 2, $M_{H}^{+}(s, q)=1$ iff $q \in R_{H}^{+}(s)$. Since $L_{2}=\left|\bigcup_{i \in N} A_{i}^{+}\right|$, then, by Lemma $3, L_{2} \geq \delta_{2}$. Therefore, by Definition $1, q \in R_{H}^{+}(s)$ implies that $\exists 1 \leq l \leq \delta_{2}$ and $\exists i_{0} \in H$ such that $M_{i_{0}}^{(l,+)}(s, q)=1$. Then the $(s, q)$ entry of matrix $G$ is $G(s, q)=1$. Therefore, $M_{H}^{+}(s, q)=$ 1 iff $G(s, q)=1$. Since $M_{H}^{+}$and $G$ are 0-1 matrices, it follows that $M_{H}^{+}=\bigvee_{t=1}^{\delta_{2}} \bigvee_{i \in H} M_{i}^{(t,+)}$.

It is now clear that joint matrices and status quo analysis matrices have the following relationship:

Theorem 3 Let $H \subseteq N . M_{H}=\bigvee_{i \in H} M_{i}^{S Q^{\left(\delta_{1}\right)}}$ and $M_{H}^{+}=\bigvee_{i \in H} M_{i}^{S Q^{\left(\delta_{2},+\right)}}$, where $\delta_{1}$ and $\delta_{2}$ denote the number of iterations required to find $R_{H}(s)$ and $R_{H}^{+}(s)$.

The aim of stability analysis is to find equilibria of a graph model that are stable for all 
DMs under appropriate stability definitions. The joint matrices $M_{H}$ and $M_{H}^{+}$are essential components for stability analysis using the matrix method [26]. Status quo analysis examines the dynamics of a conflict model and assesses whether predicted equilibria are reachable from the status quo [19]. The status quo analysis matrices $M_{i}^{S Q^{(t)}}$ and $M_{i}^{S Q^{(t,+)}}$ sufficiently depict the status quo analysis process. Thus, the two inductive procedures provided by Lemma 1 furnish a unified vehicle to carry out both stability analysis and status quo analysis using consistent and related matrix formulations in an algebraic way.

\section{Application of the Matrix Method for Status Quo Analysis}

In this section, the proposed matrix approach to status quo analysis is applied to the Elmira conflict to illustrate how the procedure works. As a small agricultural town renowned for its annual maple syrup festival, Elmira is located in southwestern Ontario, Canada. In 1989, the Ontario Ministry of Environment (MoE) detected that the underground aquifer supplying water for Elmira was polluted by N-nitroso demethylamine (NDMA). A local pesticide and rubber manufacturer, Uniroyal Chemical Ltd. (UR), was identified, since the prime suspect as NDMA is a by-product of its production line. Hence, a Control Order was issued by MoE, requiring that UR take expensive measures to remedy the contamination. UR immediately appealed to repeal this control order. The Local Government (LG) consisting of the Regional Municipality of Waterloo and the Township of Woolwich, sided with MoE and sought legal advice from independent consultants on its role to resolve this conflict (see the paper [11] for more details).

Table 1

Feasible states for the Elmira model

\begin{tabular}{|c|c|c|c|c|c|c|c|c|c|}
\hline MoE & & & & & & & & & \\
\hline 1. Modify & $\mathrm{N}$ & Y & $\mathrm{N}$ & Y & $\mathrm{N}$ & $\mathrm{Y}$ & $\mathrm{N}$ & $\mathrm{Y}$ & - \\
\hline \multicolumn{10}{|l|}{ UR } \\
\hline 2. Delay & Y & Y & $\mathrm{N}$ & $\mathrm{N}$ & Y & Y & $\mathrm{N}$ & $\mathrm{N}$ & - \\
\hline 3. Accept & $\mathrm{N}$ & $\mathrm{N}$ & Y & Y & $\mathrm{N}$ & $\mathrm{N}$ & Y & $\mathrm{Y}$ & - \\
\hline 4. Abandon & $\mathrm{N}$ & $\mathrm{N}$ & $\mathrm{N}$ & $\mathrm{N}$ & $\mathrm{N}$ & $\mathrm{N}$ & $\mathrm{N}$ & $\mathrm{N}$ & $\mathrm{Y}$ \\
\hline \multicolumn{10}{|l|}{ LG } \\
\hline 5. Insist & $\mathrm{N}$ & $\mathrm{N}$ & $\mathrm{N}$ & $\mathrm{N}$ & Y & Y & Y & $\mathrm{Y}$ & - \\
\hline State number & 1 & 2 & 3 & 4 & 5 & 6 & 7 & 8 & 9 \\
\hline
\end{tabular}

[11] established a graph model for this conflict, comprised of three DMs: 1. MoE, 2. UR, and 3. LG; and five options: 1. Modify-Modify the Control Order to make it more acceptable to UR; 2. Delay-Lengthen the appeal process; 3. Accept-Accept the cur- 


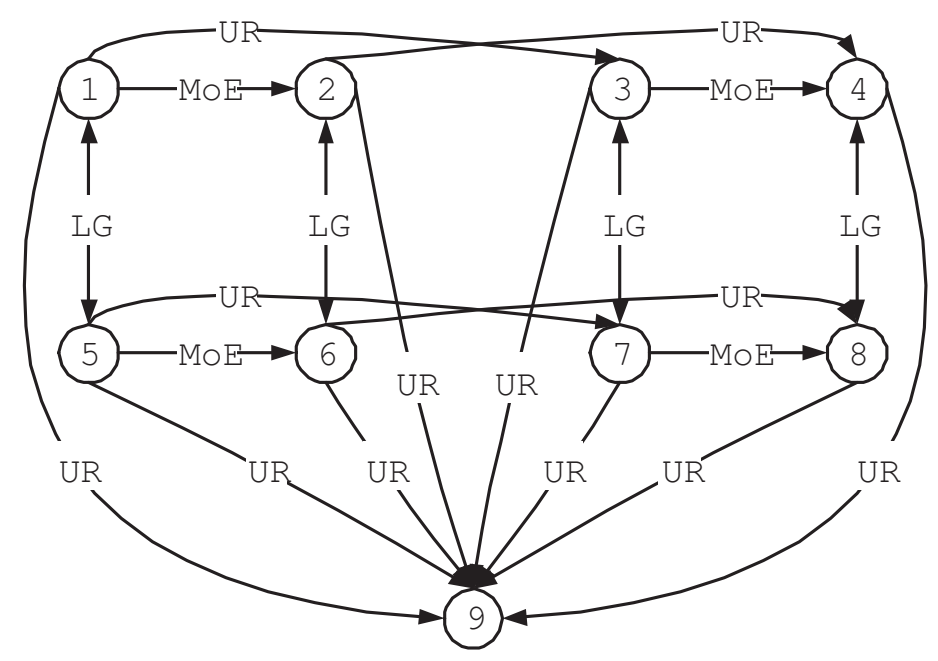

Fig. 1. A graph model for the Elmira conflict.

Table 2

Relative preferences for DMs in the Elmira conflict

\begin{tabular}{cc}
\hline DM & Preference \\
\hline $\mathrm{MoE}$ & $7 \succ 3 \succ 4 \succ 8 \succ 5 \succ 1 \succ 2 \succ 6 \succ 9$ \\
$\mathrm{UR}$ & $1 \succ 4 \succ 8 \succ 5 \succ 9 \succ 3 \succ 7 \succ 2 \succ 6$ \\
$\mathrm{LG}$ & $7 \succ 3 \succ 5 \succ 1 \succ 8 \succ 6 \succ 4 \succ 2 \succ 9$ \\
\hline
\end{tabular}

rent Control Order; 4. Abandon-Abandon its Elmira operation; and 5. Insist-Insist that the original Control Order be applied. Although there exist 32 mathematically possible states, given the five options in this model, many of them are infeasible for a variety of reasons and only 9 states are identified as being feasible and listed in Table 1 (where a "Y" indicates that an option is selected by the DM controlling it, an " $\mathrm{N}$ " means that the option is not chosen, and a dash "-" denotes that the entry may be "Y" or "N"). The graph model of the Elmira conflict is shown in Fig. 1, in which labels on the arcs indicate the DM who controls the move; and preference information over the states is given in Table 2.

Let $N=\{1,2,3\}$ be the set of three DMs $(1=\mathrm{MoE}, 2=\mathrm{UR}$, and $3=\mathrm{LG})$. To carry out status quo analysis for the Elmira model by using the matrix approach, the following steps are needed:

- Construct matrices $J_{i}$ and $P_{i}^{+}$for $i=1,2,3$, using information provided by Fig. 1 and Table 2 as well as equations (1) and (2);

- Calculate the UI matrices $J_{i}^{+}$for $i=1,2,3$, using equation (3);

- Determine the matrices $M_{i}^{(t)}$ and $M_{i}^{(t,+)}$ for $i=1,2,3$, using inductive formulations provided by Lemma 1; and

- Compute the status quo analysis matrices $M_{i}^{S Q^{(k)}}$ and $M_{i}^{S Q^{(k,+)}}$ for $i=1,2,3$, using Theorem 1. 
Table 3

Stability analysis results of the Elmira model

\begin{tabular}{|c|c|c|c|c|c|c|c|c|c|c|c|c|c|c|c|c|}
\hline State Number & \multicolumn{4}{|c|}{ Nash } & \multicolumn{4}{|c|}{ GMR } & \multicolumn{4}{c|}{ SMR } & \multicolumn{4}{c|}{ SEQ } \\
\hline & MoE & UR & LG & $E q$ & MoE & UR & LG & $E q$ & MoE & UR & LG & $E q$ & MoE & UR & LG & $E q$ \\
\hline 1 & $\sqrt{ }$ & $\sqrt{ }$ & & & $\sqrt{ }$ & $\sqrt{ }$ & $\sqrt{ }$ & $\sqrt{ }$ & $\sqrt{ }$ & $\sqrt{ }$ & $\sqrt{ }$ & $\sqrt{ }$ & $\sqrt{ }$ & $\sqrt{ }$ & & \\
\hline 2 & $\sqrt{ }$ & & & & $\sqrt{ }$ & & $\sqrt{ }$ & & $\sqrt{ }$ & & $\sqrt{ }$ & & $\sqrt{ }$ & & $\sqrt{ }$ & \\
\hline 3 & $\sqrt{ }$ & & & & $\sqrt{ }$ & & $\sqrt{ }$ & & $\sqrt{ }$ & & $\sqrt{ }$ & & $\sqrt{ }$ & & $\sqrt{ }$ & \\
\hline 4 & $\sqrt{ }$ & $\sqrt{ }$ & & & $\sqrt{ }$ & $\sqrt{ }$ & $\sqrt{ }$ & $\sqrt{ }$ & $\sqrt{ }$ & $\sqrt{ }$ & $\sqrt{ }$ & $\sqrt{ }$ & $\sqrt{ }$ & $\sqrt{ }$ & & \\
\hline 5 & $\sqrt{ }$ & $\sqrt{ }$ & $\sqrt{ }$ & $\sqrt{ }$ & $\sqrt{ }$ & $\sqrt{ }$ & $\sqrt{ }$ & $\sqrt{ }$ & $\sqrt{ }$ & $\sqrt{ }$ & $\sqrt{ }$ & $\sqrt{ }$ & $\sqrt{ }$ & $\sqrt{ }$ & $\sqrt{ }$ & $\sqrt{ }$ \\
\hline 6 & $\sqrt{ }$ & & $\sqrt{ }$ & & $\sqrt{ }$ & & $\sqrt{ }$ & & $\sqrt{ }$ & & $\sqrt{ }$ & & $\sqrt{ }$ & & $\sqrt{ }$ & \\
\hline 7 & $\sqrt{ }$ & & $\sqrt{ }$ & & $\sqrt{ }$ & & $\sqrt{ }$ & & $\sqrt{ }$ & & $\sqrt{ }$ & & $\sqrt{ }$ & & $\sqrt{ }$ & \\
\hline 8 & $\sqrt{ }$ & $\sqrt{ }$ & $\sqrt{ }$ & $\sqrt{ }$ & $\sqrt{ }$ & $\sqrt{ }$ & $\sqrt{ }$ & $\sqrt{ }$ & $\sqrt{ }$ & $\sqrt{ }$ & $\sqrt{ }$ & $\sqrt{ }$ & $\sqrt{ }$ & $\sqrt{ }$ & $\sqrt{ }$ & $\sqrt{ }$ \\
\hline 9 & $\sqrt{ }$ & $\sqrt{ }$ & $\sqrt{ }$ & $\sqrt{ }$ & $\sqrt{ }$ & $\sqrt{ }$ & $\sqrt{ }$ & $\sqrt{ }$ & $\sqrt{ }$ & $\sqrt{ }$ & $\sqrt{ }$ & $\sqrt{ }$ & $\sqrt{ }$ & $\sqrt{ }$ & $\sqrt{ }$ & $\sqrt{ }$ \\
\hline
\end{tabular}

Status quo analysis is mainly concerned with the attainability of predicted equilibria. Therefore, stability analysis is usually conducted first. Traditionally, stability analysis is performed by using the DSS GMCR II. Here, to demonstrate the effectiveness of the matrix approach, stability analyses are carried out by using the proposed methods by $\mathrm{Xu}$ et al. [26] under four solution concepts consisting of Nash stability, General Metarationality (GMR), Symmetric Metarationality (SMR), and Sequential stability (SEQ). The results are summarized in Table 3, in which " $\sqrt{ }$ " for a given state under a DM means that this state is stable for a given DM; and " $\sqrt{ }$ " for a state under " $E q$ " signifies that this state is an equilibrium for a corresponding solution concept. It is trivial to verify that the stability analysis results for the four solution concepts are identical to the findings generated by GMCR II. Table 3 identifies three states 5, 8 and 9 as strong equilibria because they are stable for all DMs under all four solution concepts.

Matrix manipulations generate the status quo analysis matrices given in Table 4 (with any UMs) and Table 5 (with UIs only). As the status quo state is 1 , we can assess the attainability of any state from the status quo state by examining its corresponding entry in the first row for each DM, where a value of 1 indicates that the associated state is reachable from the status quo state and a value of 0 means that the corresponding state is not reachable. Given the three matrices in Table 4, it is obvious that the three strong equilibria, 5, 8, and 9, are all attainable. For instance, $M_{M o E}^{S Q^{(3)}}(1,8)=1$, $M_{U R}^{S Q^{(3)}}(1,8)=1$, and $M_{L G}^{S Q^{(3)}}(1,8)=1$ demonstrate that the strong equilibrium state 8 is reachable from status quo state 1 in at most three UMs with the last mover being any of the three DMs, MoE, UR or LG. On the other hand, as the only non-zero $(1,5)$ entry of the three matrices is $M_{L G}^{S Q^{(3)}}(1,5)$, equilibrium 5 can be reached from the status quo within three UMs with LG being the unique last mover. Similarly, strong equilibrium 9 is reachable from status quo state 1 in at most three UMs with a unique last mover UR.

When only UIs are allowed as shown in Table 5, the only strong equilibrium that can be reached from the status quo state 1 is 5 , which can be achieved in at most three UIs with last mover LG, because the unique non-zero entry in the first row of the three 
Table 4

Status quo analysis matrices with unilateral moves

\begin{tabular}{c|ccccccccc|cccccccccc|ccccccccc}
\hline Matrix & \multicolumn{10}{c|}{$M_{M o E}^{S Q^{(3)}}$} & \multicolumn{1}{c|}{$M_{U R}^{S Q^{(3)}}$} & \multicolumn{1}{c}{$M_{L G}^{S Q^{(3)}}$} \\
\hline State & 1 & 2 & 3 & 4 & 5 & 6 & 7 & 8 & 9 & 1 & 2 & 3 & 4 & 5 & 6 & 7 & 8 & 9 & 1 & 2 & 3 & 4 & 5 & 6 & 7 & 8 & 9 \\
\hline 1 & 0 & 1 & 0 & 1 & 0 & 1 & 0 & 1 & 0 & 0 & 0 & 1 & 1 & 0 & 0 & 1 & 1 & 1 & 0 & 1 & 1 & 0 & 1 & 1 & 1 & 1 & 0 \\
2 & 0 & 0 & 0 & 0 & 0 & 0 & 0 & 0 & 0 & 0 & 0 & 0 & 1 & 0 & 0 & 0 & 1 & 1 & 0 & 0 & 0 & 1 & 0 & 1 & 0 & 1 & 0 \\
3 & 0 & 0 & 0 & 1 & 0 & 0 & 0 & 1 & 0 & 0 & 0 & 0 & 0 & 0 & 0 & 0 & 0 & 1 & 0 & 0 & 0 & 1 & 0 & 0 & 1 & 1 & 0 \\
4 & 0 & 0 & 0 & 0 & 0 & 0 & 0 & 0 & 0 & 0 & 0 & 0 & 0 & 0 & 0 & 0 & 0 & 1 & 0 & 0 & 0 & 0 & 0 & 0 & 0 & 1 & 0 \\
5 & 0 & 1 & 0 & 1 & 0 & 1 & 0 & 1 & 0 & 0 & 0 & 1 & 1 & 0 & 0 & 1 & 1 & 1 & 1 & 1 & 1 & 1 & 0 & 1 & 1 & 0 & 0 \\
6 & 0 & 0 & 0 & 0 & 0 & 0 & 0 & 0 & 0 & 0 & 0 & 0 & 1 & 0 & 0 & 0 & 1 & 1 & 0 & 1 & 0 & 1 & 0 & 0 & 0 & 1 & 0 \\
7 & 0 & 0 & 0 & 1 & 0 & 0 & 0 & 1 & 0 & 0 & 0 & 0 & 0 & 0 & 0 & 0 & 0 & 1 & 0 & 0 & 1 & 1 & 0 & 0 & 0 & 1 & 0 \\
8 & 0 & 0 & 0 & 0 & 0 & 0 & 0 & 0 & 0 & 0 & 0 & 0 & 0 & 0 & 0 & 0 & 0 & 1 & 0 & 0 & 0 & 1 & 0 & 0 & 0 & 0 & 0 \\
9 & 0 & 0 & 0 & 0 & 0 & 0 & 0 & 0 & 0 & 0 & 0 & 0 & 0 & 0 & 0 & 0 & 0 & 0 & 0 & 0 & 0 & 0 & 0 & 0 & 0 & 0 & 0 \\
\hline
\end{tabular}

Table 5

Status quo analysis matrices with unilateral improvements

\begin{tabular}{c|ccccccccc|cccccccccc|cccccccc}
\hline Matrix & \multicolumn{1}{c|}{$M_{M o E}^{S Q^{(3,+)}}$} & \multicolumn{1}{c|}{$M_{U R}^{S Q^{(3,+)}}$} & \multicolumn{1}{c}{$M_{L G}^{S Q^{(3,+)}}$} \\
\hline State & 1 & 2 & 3 & 4 & 5 & 6 & 7 & 8 & 9 & 1 & 2 & 3 & 4 & 5 & 6 & 7 & 8 & 9 & 1 & 2 & 3 & 4 & 5 & 6 & 7 & 8 & 9 \\
\hline 1 & 0 & 0 & 0 & 0 & 0 & 0 & 0 & 0 & 0 & 0 & 0 & 0 & 0 & 0 & 0 & 0 & 0 & 0 & 0 & 0 & 0 & 0 & 1 & 0 & 0 & 0 & 0 \\
2 & 0 & 0 & 0 & 0 & 0 & 0 & 0 & 0 & 0 & 0 & 0 & 0 & 1 & 0 & 0 & 0 & 1 & 1 & 0 & 0 & 0 & 0 & 0 & 1 & 0 & 1 & 0 \\
3 & 0 & 0 & 0 & 0 & 0 & 0 & 0 & 0 & 0 & 0 & 0 & 0 & 0 & 0 & 0 & 0 & 0 & 1 & 0 & 0 & 0 & 0 & 0 & 0 & 1 & 0 & 0 \\
4 & 0 & 0 & 0 & 0 & 0 & 0 & 0 & 0 & 0 & 0 & 0 & 0 & 0 & 0 & 0 & 0 & 0 & 0 & 0 & 0 & 0 & 0 & 0 & 0 & 0 & 1 & 0 \\
5 & 0 & 0 & 0 & 0 & 0 & 0 & 0 & 0 & 0 & 0 & 0 & 0 & 0 & 0 & 0 & 0 & 0 & 0 & 0 & 0 & 0 & 0 & 0 & 0 & 0 & 0 & 0 \\
6 & 0 & 0 & 0 & 0 & 0 & 0 & 0 & 0 & 0 & 0 & 0 & 0 & 0 & 0 & 0 & 0 & 1 & 1 & 0 & 0 & 0 & 0 & 0 & 0 & 0 & 0 & 0 \\
7 & 0 & 0 & 0 & 0 & 0 & 0 & 0 & 0 & 0 & 0 & 0 & 0 & 0 & 0 & 0 & 0 & 0 & 1 & 0 & 0 & 0 & 0 & 0 & 0 & 0 & 0 & 0 \\
8 & 0 & 0 & 0 & 0 & 0 & 0 & 0 & 0 & 0 & 0 & 0 & 0 & 0 & 0 & 0 & 0 & 0 & 0 & 0 & 0 & 0 & 0 & 0 & 0 & 0 & 0 & 0 \\
9 & 0 & 0 & 0 & 0 & 0 & 0 & 0 & 0 & 0 & 0 & 0 & 0 & 0 & 0 & 0 & 0 & 0 & 0 & 0 & 0 & 0 & 0 & 0 & 0 & 0 & 0 & 0 \\
\hline
\end{tabular}

matrices is $M_{L G}^{S Q^{(3,+)}}(1,5)$.

If a different state is selected as the status quo state, one can conveniently examine the elements of the corresponding row in the relevant status quo analysis matrices to evaluate the attainability of any state that is of interests.

Table 6

The results of status quo analysis for the the Elmira conflict

\begin{tabular}{|c|c|c|c|c|c||c|c|c|c|c|c|}
\hline State & $V^{(0)}$ & $V^{(1)}$ & $V^{(2)}$ & $V^{(3)}$ & $V^{(4)}$ & State & $V^{(0,+)}$ & $V^{(1,+)}$ & $V^{(2,+)}$ & $V^{(3,+)}$ & $V^{(4,+)}$ \\
\hline 1 & $\sqrt{ }$ & & & & & 2 & $\sqrt{ }$ & & & & \\
\hline 2 & & 1 & 1 & 1,3 & 1,3 & 4 & & 2 & 2 & 2 & 2 \\
\hline 3 & & 2 & 2 & 2,3 & 2,3 & 6 & & 3 & 3 & 3 & 3 \\
\hline 5 & & 3 & 3 & 3 & 3 & 9 & & 2 & 2,3 & 2,3 & 2,3 \\
\hline 9 & & 2 & 2 & 2 & 2 & 8 & & & 2 & 2 & 2 \\
\hline 4 & & & 1,2 & 1,2 & 1,2 & 1 & & & & & \\
\hline 6 & & & 1,3 & 1,3 & 1,3 & 3 & & & & & \\
\hline 7 & & & 2,3 & 2,3 & 2,3 & 5 & & & & & \\
\hline 8 & & & & $1,2,3$ & $1,2,3$ & 7 & & & & & \\
\hline
\end{tabular}

By using the proposed inductive formulations in Theorem 1, the status quo analysis 
result can also be presented in a tableau form as shown in Table 6. Note that in Table 6 , state $1(\sqrt{ })$ and state $2(\sqrt{ })$ are sequentially selected as the status quo state with any UMs and UI, respectively. It is easy to verify the equivalence of the results here and those given by Li et al. [19], except for the difference in recording the last mover information in the two approaches. This table offers a wealth of information, such as the specific $\operatorname{DM}(\mathrm{s})$ as the last mover(s) and the shortest path(s) to reach a state. For example, the shortest path to strong equilibrium 8 from status quo state 1 requires three legal UMs with any of the three DMs being the last mover. However, if state 2 is selected as the status quo, then equilibrium 8 is reachable with the shortest path of two UMs and a unique last mover DM 2.

By taking a status quo analysis into account, additional insights are revealed about the attainability of any potential resolution and, if attainable, the dynamics of conflict evolution from the status quo state is demonstrated. The results offered by Table 6 are identical to those provided by Li et al. [19].

\section{Conclusions}

Status quo analysis aims to look forward from the status quo for identifying attainable states and assessing how readily they can be reached. Although status quo analysis was developed early in the graph model, and GMCR II provides some basic analysis for the relevance of the status quo, the concept was not sufficiently developed to include in the decision support system. The new developments in status quo analysis and corresponding pseudo-codes are introduced by $\mathrm{Li}$ et al. $[18,19]$, but they have not been implemented using computers so far.

In this paper, a matrix representation is developed for conducting status quo analysis in the graph model for conflict resolution. Due to the nature of the explicit algebraic expressions, the matrix method has advantages of convenient computer implementation and easy expansion to new analysis techniques. Consequently, it is possible to extend the matrix method to the graph model with uncertain preference for status quo analysis [20]. The relationship between the joint matrices and status quo analysis matrices provided by Theorem 3 demonstrates that the computational complexity of calculating the status quo analysis matrices is less than that of constructing the joint matrices which presents a polynomial-time effective algorithm analyzed by Xu et al. [26]. Therefore, the proposed method has the potential to deal with large and complicated conflict models. Inherently, the proposed approach is based on the adjacency matrix to search state-by-state paths. If a graph model contains multiple arcs between the same two states controlled by different DMs, the state-by-state paths will not be able to track all aspects of the evolution of a conflict from the status quo state, and an expanded model will be needed to allow for searching arc-by-arc paths.

Furthermore, an important restriction in a graph model is that no DM can move twice in 
succession along any path. If different DMs are denoted by distinct colors, then tracing the evolution of a conflict requires searching all paths in the integrated graph that start from the status quo state and do not contain consecutive arcs with the same color. In this case, it is difficult to use existing methods or algorithms directly, including the genetic algorithm [4] and the neural network [23], to find the paths, due to the additional feature of color restrictions that is not present in existing problems. The proposed method furnishes a potential tool for designing a conversion function to transform the original problem of searching paths with certain practical constraints to a standard problem of finding paths without such restrictions so that existing methodologies can be easily adapted for use in practice. These open problems warrant further investigations and will be discussed in future research.

\section{References}

[1] P.G. Bennett, "Modelling decision in international relations: game theory and beyond," Mershon International Studies Review, 39 (1995), 19-52.

[2] J.W. Burton, "Conflict resolution as a political philosophy," In: D.J.D. Sandole and H. van der Merwe (Eds), Conflict Resolution Theory and Practice: Integration and Application, 5564, Manchester: Manchester University Press, 1993.

[3] J. Burton and F. Dukes, Conflict: Practices in Management, Settlement, and Resolution. New York: St. Martin's Press, 1990.

[4] C. Davies and P. Lingras, "Genetic algorithms for rerouting shortest paths in dynamic and stochastic networks", European Journal of Operational Research 144 (2003) 27-38.

[5] E. De Bono, Conflicts: A Better Way to Resolve Them. London: Harrap, 1985.

[6] M. Deutsch, The Resolution of Conflict: Constructive and Destructive Processes, New Haven: Yale University Press, 1973.

[7] L. Fang, K.W. Hipel, and D.M. Kilgour, Interactive Decision Making: The Graph Model for Conflict Resolution. New York: Wiley, 1993.

[8] L. Fang, K.W. Hipel, D.M. Kilgour, and X. Peng, "A decision support system for interactive decision making, Part 1: Model formulation," IEEE Transactions on Systems, Man and Cybernetics, Part C 33 (1) (2003), 42-55.

[9] L. Fang, K.W. Hipel, D.M. Kilgour, and X. Peng, "A decision support system for interactive decision making, Part 2: Analysis and output interpretation", IEEE Transactions on Systems, Man and Cybernetics, Part C 33 (1) (2003), 56-66.

[10] L. Hamouda, D.M. Kilgour, and K.W. Hipel, "Strength of preference in graph models for multiple-decision-maker conflicts", Applied Mathematics and Computation 179 (2006), 314-327.

[11] K.W. Hipel, L. Fang, D.M. Kilgour, and M. Haight, "Environmental conflict resolution using the graph model", Proceedings IEEE SMC International Conference 1 (1993), 153-158. 
[12] K.W. Hipel, D.M. Kilgour, L. Fang, and X. Peng, "The decision support system GMCR II in environmental conflict management", Applied Mathematics and Computation 83 (2 and 3) (1997), 117-152.

[13] K.W. Hipel, "Conflict resolution: Theme overview paper in conflict resolution, in Encyclopedia of Life Support Systems (EOLSS), Oxford, U.K.: EOLSS Publishers, 2002.

[14] N. Howard, Confrontation Analysis: How to Win Operations Other Than War. The Pentagon, Washington D.C.: DoD C4ISR Cooperative Research Program, 1999.

[15] N. Howard, P.G. Bennett, J.W. Bryant, and M. Bradley, "Manifesto for a theory of drama and irrational choice," Journal of the Operational Research Society 44 (1992), 99-103.

[16] D.M. Kilgour, K.W. Hipel, and L. Fang, "The graph model for conflicts", Automatica 23 (1987), 41-55.

[17] K.W. Li, K.W. Hipel, D.M. Kilgour, and L. Fang, "Preference uncertainty in the graph model for conflict resolution", IEEE Transactions on Systems, Man and Cybernetics, Part A: Systems and Humans 34 (2004), 507-520.

[18] K.W. Li, D.M. Kilgour, and K.W. Hipel, "Status quo analysis of the Flathead river conflict", Water Resources Research 40 (2004), W05S03.

[19] K.W. Li, D.M. Kilgour, and K.W. Hipel, "Status quo analysis in the graph model for conflict resolution", Journal of the Operational Research Society 56 (2005), 699-707.

[20] K.W. Li, K.W. Hipel, D.M. Kilgour, and D.J. Noakes, "Integrating uncertain preference into status quo analysis with applications to an environmental conflict", Group Decision and Negotiation 14 (2005), 461-479.

[21] T.L. Saaty and J.M. Alexander, Conflict Resolution: The Analytic Hierarchy Approach. New York: Praeger Publishers, 1989.

[22] L.C. Thomas, "Using Game Theory and its Extensions to Model Conflict". In: P.G. Bennett (Ed.), Analyzing Conflict and Its Resolution: Some Mathematical Contributions, New York: Oxford University Press, 1987.

[23] Y. Xia and J. Wang, "A discrete-time recurrent neural network for shortest-path routing", IEEE Transactions on Automatic Control 45(11) (2000), 2129-2134.

[24] H. Xu, K.W. Hipel, and D.M. Kilgour, "Matrix representation of conflicts with two decision-makers", IEEE 2007 International Conference on Systems, Man and Cybernetics 1 (2007), 1764-1769.

[25] H. Xu, D.M. Kilgour., and K.W. Hipel, "Matrix representation of solution concepts in graph models for two decision-makers with preference uncertainty", Dynamics of Continuous, Discrete and Impulsive Systems, A Supplement 14 (S1) (2007), 703-707.

[26] H. Xu, K.W. Hipel, and D.M. Kilgour, "Matrix representation of solution concepts in multiple decision maker graph models", IEEE Transactions on Systems, Man, and Cybernetics-Part A 39 (1) (2009), 96-108.

[27] M. Wang, K.W. Hipel, and N.M. Fraser, "Solution concepts in hypergames", Applied Mathematics and Computation 34 (1989), 147-171. 
Response to the referee's comments

on the paper AMC-D-08-00620

\title{
"A Matrix Approach to Status Quo Analysis in the Graph Model for
} Conflict Resolution"

by

\author{
Haiyan Xu, Kevin W. Li, Keith W. Hipel, and D. Marc Kilgour \\ Submitted for publication in the \\ Applied Mathematics and Computation
}

February 13, 2009

We would like to thank you for carefully reviewing our paper and providing useful comments to enhance its quality. Our revisions, written in response to your comments, are explained below.

- It is recommended to the authors to maybe expand on the limitations and advantages of their model as compared to other existent methodologies not only from a mathematical angle but also from its applicability and relevance to Conflict Resolution.

Following the referee's thoughtful suggestion, Section 5 has been significantly expanded to address the limitations and advantages of the proposed approach from both a mathematical angle and its applicability and relevance to conflict resolution perspective. More specifically, the second paragraph in Section 5 has been expanded to comment on the advantages and limitations of the proposed approach here and a new third paragraph has been added to lay out a framework for future research to enhance the applicability of this model.

- Page 2: Second paragraph Recently NOT recently and Page 3: First paragraph last line double "the"

These typos have been corrected in this revision.

- Page 3: the asymmetric $a \succeq_{i} b$ in the fourth line of Section 2.1 has an equal line below that does not appear in any of the others. Is that an error?

This notation is introduced to facilitate defining the function $p_{i}(s)$ in Equation (1) on page 4.

- Page 4: in equation 1, q has not been defined and "|.|" denotes the number of states but was never used in equation 1 so does it go here?

The description of Equation (1) on page 4 has been slightly modified to clarify this definition.

- the order of (iii) and (v) makes it confusing, if possible I would move (v) to become (iv) for better readability

(iii) and (v) have now been combined as per the referee's suggestion. (see page 5) 
- Page 6: what is Pi(.)?... it was never defined in 2.1

The notation $p_{i}($.$) has been changed to p_{i}(s)$ as defined in Section 2.1.

- Page 7: How is "|.|" from here related to the one in page 4?

In order to avoid confusion, the expression in equation (2) on page 7 has been modified with an appropriate explanation.

- Page 7: last paragraph, should this be $N$ rather than $n$ ?

In the last paragraph on page $7, n$ is correct. To make it clear, a definition of $n$ is introduced therein: "Let $n=|N|$ denote the number of DMs in the set $N$."

- Page 8: in Pseudo-code I would tab to the left the otherwise so that it aligns with the if above.. same on Page 9

The Pseudo-codes on page 8 and page 9 have been reconfirmed.

- Page 10: Just to be sure, should DMi on $Q(s, q)$ be $i$ or $j$ ?

It is indeed DM $i$ in $Q(s, q)$ and this $Q$ serves as an intermediate variable in the calculation process. 